\title{
Precise Calculations of Atomic Electron Binding Energies in Fermium*
}

\author{
B. Fricke, J. P. Desclaux, $\dagger$ and J. T. Waber \\ Department of Material Sciences, Northwestern University, Evanston, Ilinois 60201
}

(Received 10 January 1972)

\begin{abstract}
The comparison between the experimental binding energies for the $K, L$, and $M$ electrons for fermium and the results of our Dirac-Fock calculation, taking into account all tractable corrections, leads to agreement within about $20 \mathrm{eV}$. This shows that the present method of calculation is an adequate description of this problem and that nonlinear electrodynamical effects will not be present in nature or will be smaller than $1 \%$ compared to the values recently proposed. It is found that the energies of electronic transitions can now be used to estimate the nuclear radius.
\end{abstract}

During the last year the energies of the $K \alpha \mathrm{x}-$ ray lines of the very high- $Z$ element ${ }^{251} \mathrm{Fm}$ were obtained by Dittner et al. ${ }^{1}$ with an experimental error of about $90 \mathrm{eV}$. A short time later Porter and Freedman ${ }^{2}$ measured the atomic electron binding energies for the inner shells of ${ }^{254} \mathrm{Fm}$ with conversion electron spectroscopy and an experimental error of only about $10 \mathrm{eV}$. This precision is much better than the accuracy any $\mathrm{Di}-$ rac-Slater atomic calculation can ever obtain because of the approximation used for the exchange potential. Besides this principal uncertainty which makes the use of Dirac-Fock calculations inevitable, a number of additional corrections have to be taken into account. The magnetic interaction and the retardation as well as the quantum-electrodynamical effects-vacuum fluctuation and vacuum polarization-become more and more important with increasing $Z$. Their contribution to the binding energy of the $1 \mathrm{~s}$ electrons in fermium is already as large as $1 \mathrm{keV}$. Hence they must be known precisely.

A comparison between the experimental results and the calculations for very high- $Z$ elements such as fermium becomes very important, because two statements can be made if close agreement is achieved. First, the self-consistentfield Dirac-Fock description is a good description at least for the inner shells of the atom; and second, the present calculations of the corrections are sufficient as well. These two statements are not trivial for two reasons. First, from the newest precision measurements of radiative transitions in muonic atoms made by Dixit et al. ${ }^{3}$ it has been learned that a small discrepancy is still observed between conventional quantum electrodynamics and experiment, even after taking into account all the corrections which are tractable up to higher order. Second, one expects a large difference between the Dirac-Fock calculations and the experiment for superheavy elements near the second island of stability, ${ }^{4}$ which is near $Z=164$. This is because strong quantum-electrodynamical effects ${ }^{5}$ are one of the explanations for why the electron binding energies are not expected to reach the negative continuum of the electrons. Another possible explanation is nonlinear electrodynamical effects, as proposed by Rafelski, Fulcher, and Greiner, ${ }^{6}$ which were based on a modification of the BornInfeld theory.' The latter explanation should not only lead to great changes of the binding energies of the inner electrons of superheavy elements, but should also lead to effects which are observable in very heavy normal atoms.

Mann and Johnson ${ }^{8}$ have recently compared the experimental results for the $1 \mathrm{~s}$ binding energies of $\mathrm{W}, \mathrm{Hg}, \mathrm{Pb}$, and $\mathrm{Rn}$ with Dirac- Fock calculations which took into account all tractable corrections. They found very good agreement. But still the atomic number was not high enough to get an accurate test for the nonlinear electrodynamical contribution. Rafelski, Fulcher, and Greine ${ }^{6}$ claim a dependence of this effect of about $Z^{10}$ in this area of elements. Therefore, we have made accurate Dirac-Fock calculations ${ }^{9}$ plus estimations and calculations of the corrections for the $K-, L-$, and two of the $M$-shell electrons for fermium. The results are given in Table I.

In row 1 the differences are given between the total energies of the neutral atom and the atom with a hole in the inner shell. These numbers therefore include already the rearrangement effect which is $117 \mathrm{eV}$ for the $1 \mathrm{~s}$ electron binding energy, about $75 \mathrm{eV}$ for the electrons of the $L$ shell, and about $45 \mathrm{eV}$ for the $M$ shell.

Fermium is not a closed-shell atom. Except for the $5 f_{7 / 2}$ subshell, the neutral fermium atom contains only closed shells. Hence, only the exchange part of the Breit interaction is nonvanishing for these. For the whole state of the $1 S_{1 / 2}$, 
TABLE I. Experimental Dirac-Fock inner-electron binding energies (in keV) for fermium, plus all known corrections.

\begin{tabular}{|c|c|c|c|c|c|c|}
\hline Source & $1 s$ & $2 s$ & $2 p_{1 / 2}$ & $2 p_{3 / 2}$ & $3 s$ & $3 p_{1 / 2}$ \\
\hline Electric & -142.929 & -27.734 & -26.791 & -20.947 & -7.250 & -6.815 \\
\hline Magnetic & +0.715 & +0.091 & +0.153 & +0.092 & +0.019 & +0.033 \\
\hline Retardation & -0.041 & -0.008 & -0.013 & -0.011 & -0.001 & -0.003 \\
\hline Vacuum fluctuation & +0.457 & +0.096 & +0.009 & -0.003 & +0.025 & +0.003 \\
\hline Vacuum polarization & -0.155 & -0.026 & -0.004 & +0.000 & -0.006 & -0.001 \\
\hline Theoretical total & $-141.953(26)$ & $-27.581(20)$ & $-26.646(10)$ & $-20.869(10)$ & $-7.213(15)$ & $-6.783(4)$ \\
\hline Experimental value $^{a}$ & $-141.963(13)$ & $-27.573(8)$ & $-26.664(7)$ & $-20.868(7)$ & $-7.200(9)$ & $-6.779(7)$ \\
\hline
\end{tabular}

${ }^{\text {a }}$ Ref. 2 .

$2 S_{1 / 2}$, and $2 P_{1 / 2}$ shells of the ion, there is a direct contribution from the interaction of the single orbital with the closed shells. The present DiracFock program treats this interaction correctly. The remaining small part, namely, the direct magnetic interaction between the electron in the open $5 f_{7 / 2}$ subshell was calculated, but was found to be trival when compared with the other energies under consideration. Further, our calculations of the magnetic terms agree well with those of Mann and Johnson, ${ }^{8}$ where they can be compared. We have also calculated the contribution to the total energy for different angular momenta in the neutral atom and the atoms with the hole in the inner shell. We found that this contribution is less than $10^{-2}$ Ry in all cases so that the differences between the average total energies as given in row 1 are fully sufficient at this time.

Row 2 contains the differences of the total magnetic energies between the neutral atom and the ion with the hole. This contribution is calculated by perturbation theory (classically speaking this is an expansion in $v / c$ ), which is the proper treatment. Up to now, any higher-order contribution to this interaction is unknown, but it might be responsible for a small discrepancy in the $1 \mathrm{~s}$ binding energy because the ratio of its orbital velocity $v / c$ is already of the order of 0.6 .

In row 3 we list the retardation corrections taken from an extrapolation of the values given by Mann and Johnson. ${ }^{8}$

Row 4 contains our vacuum-fluctuation contribution. Desiderio and Johnson ${ }^{10}$ made accurate calculations of this effect for the $1 s$ states of the elements $Z=70$ to 90 according to a method developed by Brown et al. ${ }^{11}$ Similar but less-accurate values, for elements beyond $Z=90$, were also given by Erickson. ${ }^{12}$ We took our value only for the $1 s$ binding energy from an extrapolation of the results of Desiderio and Johnson. ${ }^{10}$ They made their calculations with Coulomb wave functions, and in addition they assumed a screening correction of about $2 \%$. During the calculations of the vacuum polarization correction, we found that for an atom like fermium the screening correction will be at least on the order of $9 \%$, which is the screening correction we used. We were able to calculate the values for the vacuum-fluctuation contribution for other electrons than those in the $1 s$ shell using a phenomenological method. ${ }^{13}$ This method might not be too accurate, but we have shown that it will give the right order of magnitude.

In row 5 the vacuum-polarization correction is listed. We have calculated this effect by introducing the exact first-order vacuum-polarization potential ${ }^{14}$ into the Hamiltonian. This treatment is equivalent to an exact higher-order perturbation calculation of the atomic wave function. To be consistent, we also took into account the next higher-order vacuum-polarization corrections as given by Fricke. ${ }^{14}$ However, these higher effects lead to a significant contribution for only the $1 s$ state; it is of the order of $3 \mathrm{eV}$.

We did not take into account any calculation of the correlation energy, first, because it will be equal or smaller than $1 \mathrm{eV}$, and second, because there exist only very rough estimations of this effect for the inner shells.

The result of this series of calculations for fermium are compared with the binding energies, measured by Porter and Freedman, ${ }^{2}$ in the last two rows. The uncertainties of the calculations are considered to be of the order of $\frac{1}{2}$ to 2 Ry depending on the level.

Up to now we have not discussed the effect of the extended nucleus on the binding energies. We assumed in our calculations a Fermi-type nucle- 
ar charge distribution with a half-density radius $C=7.0 \mathrm{fm}$ and a surface thickness $t=2.2 \mathrm{fm}$ which we extrapolated from a continuation of the experimental values given in Landolt-Börnstein. ${ }^{15} \mathrm{Al}-$ ready a change of $\Delta C=0.1 \mathrm{fm}$ changes the $1 \mathrm{~s}$ binding energy by nearly $8 \mathrm{eV}$. Thus if we knew all other corrections exactly we would be able to limit the nuclear radius for fermium with the present experimental errors within $\pm 0.2 \mathrm{fm}$. Nevertheless, with the given error bars we find the nuclear radius to be between 6.7 and $7.3 \mathrm{fm}$ which is the first example of determining a nuclear radius with the help of normal electronic atoms. Small differences may of course arise for measurements of different isotopes such as ${ }^{251} \mathrm{Fm}$ measured by Dittner et al. ${ }^{1}$ and ${ }^{254} \mathrm{Fm}$ measured by Porter and Freedman. ${ }^{2}$

The agreement between the calculated and experimental values is well within the given accuracy for all levels. But the largest differences arise for the $1 s, 2 s$, and $3 s$ levels. These are the levels with large vacuum-fluctuation corretions. Since this correction is the one with the largest error, we think that we may use these differences to determine "experimental" values for the vacuum-fluctuation contribution which will be about $100 \mathrm{eV}$ for the $2 \mathrm{~s}$ level and $30 \mathrm{eV}$ for the $3 s$ level.

This discussion of the binding ene rgies of the inner electrons for fermium shows the following: (1) It indicates that the Dirac-Fock self-consistent-field description is still a good description for the inner electrons of such a very heavy element like fermium. (2) The calculations of the various corrections are fairly good. Of course, some work has to be done, especially for the higher-order contributions to all corrections. (3) No unexplained discrepancy occurs, which is a strong indication that nonlinear electrodynamical effects are not important. If they do exist in nature they will be smaller than $1 \%$ of the pro- posed magnitude in fermium. (4) This indicates that with the present methods we are able to calculate accurate $K, L$, and $M$ x-ray transition energies for superheavy elements on the expected island of stability near $Z=114$, which may be used to discover and confirm the existence of these elements.

*Work supported by the U. S. Atomic Energy Commission.

ton leave of absence from Commissariat a l'Energie Atomique C.E.L., 94 Villeneuve St.-Georges, France.

'P. F. Dittner, C. E. Bemis, D. C. Hansley, R. J. Silva, and C. D. Goodman, Phys. Rev. Lett. 26, 1037 (1971).

${ }^{2}$ F. T. Porter and M. S. Freedman, Phys. Rev. Lett. 27,293 (1971).

${ }^{3}$ M. S. Dixit, H. L. Anderson, C. K. Hargrove, R. J, McKee, D. Kessler, H. Mes, and A. C. Thompson, Phys. Rev. Lett. 27, 878 (1971).

${ }^{4} \mathrm{~W}$. Greiner, in Proceedings of the Intermational Conference on Properties of Nuclear States, Montreal, Canada, 1969, edited by M. Harvey et al. (Presses de 1'Université de Montréal, Montréal, Canada, 1969).

${ }^{5} \mathrm{~B}$. Fricke and J. T. Waber, to be published.

${ }^{6}$ J. Rafelski, L. P. Fulcher, and W. Greiner, Phys. Rev. Lett. 27, 958 (1971).

${ }^{7}$ M. Born and L. Infeld, Proc. Roy. Soc., Ser. A 144, 425 (1934).

${ }^{8}$ J. B. Mann and W. R. Johnson, Phys. Rev. A $\underline{4}, 41$ (1971).

${ }^{9} \mathrm{~J}$. P. Desclaux, to be published.

${ }^{10}$ A. M. Desiderio and W. R. Johnson, Phys. Rev. A $\underline{3}$, $1267(1971)$

${ }^{11}$ G. E. Brown, J. S. Langer, and G. W. Schaefer, Proc. Roy. Soc., Ser. A 251, 92 (1959); G. E. Brown and D. F. Mayers, Proc. Roy. Soc., Ser. A 251, 105 (1959).

${ }^{12}$ G. W. Erickson, Phys. Rev. Lett. 27, 780 (1971).

${ }^{13}$ B. Fricke, Lett. Nuovo Cimento 2, 293 (1971).

${ }^{14} \mathrm{~B}$. Fricke, Z. Phys. 218, 495 (1969).

${ }^{15}$ Landolt-Bömstein: Numerical Data and Functional Relationships in Science and Technology, edited by H. R. Collard, L. R. B. Elton, R. Hofstadter, and H. Schopper (Springer, Berlin, 1967), Group I, Vol. 2. 